\title{
Lesões aftoides da cavidade oral: apresentação clínica, diagnóstico e tratamento
}

\author{
Aphthous lesions of the oral cavity: clinical presentation, diagnosis and treatment \\ Lesiones aftosas de la cavidad bucal: presentación clínica, diagnóstico y tratamiento
}

Áquila de Oliveira Afonso

ORCID: https://orcid.org/0000-0003-2392-4022

Universidade Evangélica de Goiás, Brasil

E-mail: aquilaafonso1@gmail.com

Elenice de Fatima Souza Capelario

ORCID: https://orcid.org/0000-0001-6460-9593

Centro Universitário UniBrasil, Brasil

E-mail: odonto.elenice@gmail.com

Francisco Andesson Bezerra da Silva

ORCID: https://orcid.org/0000-0001-9957-612X

Secretaria do Estado da Saúde, Brasil

Universidade Católica de Santos, Brasil

E-mail: andessonbr@hotmail.com

Mateus De Lima Santos

ORCID: https://orcid.org/0000-0002-3315-7034

Universidade CEUMA, Brasil

E-mail: mateusmedicina123@gmail.com

Luis Fernando Alves Correa

ORCID: https://orcid.org/0000-0001-7375-1751

Universidade Evangélica de Goiás, Brasil

E-mail: luis.correa@aluno.unievangelica.edu.br

Bianca Thuany Maciel

ORCID: https://orcid.org/0000-0003-4869-7022

C.E.O.F.E Malaquias, Brasil

E-mail: bianca.btm@hotmail.com

Geovana Martins Lopes

ORCID: https://orcid.org/0000-0001-8993-3648

Centro Universitário do Norte Paulista, Brasil

E-mail: gemartinslopes@gmail.com

Danielle da Paixão Freitas

ORCID: https://orcid.org/0000-0002-5274-5026

Centro Universitário Presidente Tancredo de Almeida Neves, Brasil

E-mail: freitas_danielle@yahoo.com

Ilana Francisca de Sousa Araújo Albuquerque

ORCID: https://orcid.org/0000-0002-0372-0615

Universidade CEUMA, Brasil

E-mail: enf_ilana@hotmail.com

Raynara Brito Silva

ORCID: https://orcid.org/0000-0002-8002-6192 Universidade CEUMA, Brasil

E-mail: raynara.silva180@gmail.com

Vittor Dorinato de Santana Sátiro

ORCID: https://orcid.org/0000-0002-7069-0807 Universidade Paulista, Brasil

E-mail: vittorodonto@gmail.com

Ronize Peruzzi Damião

ORCID: https://orcid.org/0000-0002-3308-957X Universidade Regional Integrada do Alto Uruguai e das Missões, Brasil

E-mail: ronizedamiao20@ hotmail.com

Amanda Gonçalves Franco

ORCID: https://orcid.org/0000-0003-0983-7539 Universidade de Itaúna, Brasil

E-mail: amandagfranco38@gmail.com

Lucas Dantas Pereira

ORCID: https://orcid.org/0000-0002-1551-5264

Universidade do Estado do Rio Grande do Norte, Brasil

E-mail: lucascaico22@gmail.com

Lays Guarnieri Teixeira da Silva

ORCID: https://orcid.org/0000-0001-7081-0410

Universidade Estácio de Sá, Brasil

E-mail: laysguarnieri@hotmail.com 
Tiago Pedro Pinto de Souza

ORCID: https://orcid.org/0000-0002-3853-3801 Centro Universitário UNIFACISA, Brasil

E-mail: tiagopinto6565@gmail.com

Anne Caroline Silva Freire de Sá

ORCID: https://orcid.org/0000-0003-3860-1373 Centro Universitário Tabosa de Almeida, Brasil

E-mail: annecarolinefreiree@gmail.com

Harysson Costa Melo

ORCID: https://orcid.org/0000-0001-7427-7975

Universidade de Fortaleza, Brasil

E-mail: haryssoncoslo@gmail.com

\begin{abstract}
Resumo
As lesões aftoides compreendem um grupo de doenças caracterizadas por ulcerações superficiais, dolorosas, únicas ou múltiplas, localizadas na mucosa oral. A lesão aftoide típica é pequena, redonda ou ovóide, com margens circunscritas, halo eritematoso e fundo cinzento ou amarelado. Esse estudo objetivou revisar a literatura acerca lesões aftoides da mucosa oral, destacando sua apresentação clínica, diagnóstico e tratamento, bem como a importância de um correto manejo clínico em pacientes que possuem tais lesões na cavidade oral. Para a construção deste trabalho foi realizado um levantamento bibliográfico nas bases de dados SciVerse Scopus, Scientific Eletronic Library Online (Scielo), U.S. National Library of Medicine (PUBMED) e ScienceDirect, utilizando o gerenciador de referências Mendeley. Estudos científicos disponíveis na literatura relatam que há diversas lesões aftoides que acometem a cavidade oral. Essas doenças envolvem a estomatite aftosa recorrente, doença de Behçet, Lúpus eritematoso sistêmico, mucosite e queimadura química. As lesões podem prejudicar a qualidade de vida do paciente que apresentam queixas de desconforto proveniente dessas feridas. Portanto, torna-se imprescindível que os profissionais da saúde estejam familiarizados com essas desordens, uma vez que as lesões orais podem servir como indicadoras fundamentais no diagnóstico precoce da doença.
\end{abstract}

Palavras-chave: Lesões aftoides; Etiologia; Tratamento; Diagnóstico.

\begin{abstract}
Aphthoid lesions comprise a group of diseases characterized by single or multiple, painful, superficial ulcerations located on the oral mucosa. A typical aphthoid lesion is small, round or ovoid, with circumscribed margins, erythematous halo, and a pale or yellowish background. This study aimed to review the literature on aphthoid lesions of the oral mucosa, highlighting their clinical presentation, diagnosis and treatment, as well as the importance of a correct clinical management in patients who have such lesions in the oral cavity. For the construction of this work, a bibliographic survey was carried out in the databases SciVerse Scopus, Scientific Electronic Library Online (Scielo), U.S. National Library of Medicine (PUBMED) and ScienceDirect, using the Mendeley reference manager. Scientific studies available in the literature report that there are various aphthoid lesions that affect the oral cavity. These cases involve recurrent aphthous stomatitis, Behçet's disease, systemic lupus erythematosus, mucosite and chemical burn. These injuries can harm the quality of life of patients who present complaints of discomfort from these injuries. Therefore, it becomes essential that health professionals are familiar with these disorders, once these oral lesions can serve as fundamental indicators of early diagnosis of disease.
\end{abstract}

Keywords: Aphthoid lesions; Etiology; Treatment; Diagnosis.

\title{
Resumen
}

Como lesões aftóides comprenden un grupo de doenças caracterizadas por ulcerações superficiales, dolorosas, únicas o múltiples, localizadas en la mucosa oral. Una lesión típica es pequeña, redonda o ovóide, con márgenes circunscritos, halo eritematoso e fundo cinzento o amarelado. Esse estudo objetivou review a literatura about lesões aftóides da mucosa oral, destacando sua apresentação clinic, diagnostique e tratamento, bem as a importância de um correto manejo clínico empatients that possuem tais lesões na cavidade oral. Para la construcción de este trabajo para realizar un levantamiento bibliográfico a partir de las bases de datos SciVerse Scopus, Scientific Eletronic Library Online (Scielo), U.S. National Library of Medicine (PUBMED) y ScienceDirect, utilizando el gestor de referencias Mendeley. Estudos científicos disponíveis na literatura relatam que há diversas lesões aftóides que acometem a cavidade oral. Essas doenças envolvem a estomatite aftosa recorrente, doença de Behçet, Lúpus eritematoso sistêmico, mucosite e queimadura química. Como les puede perjudicar la calidad de vida del paciente que apresentam queixas de desconforto provienen dessas feridas. Portanto, torna-se imprescindível que os profissionais da saúde estejam se conocen com essas desordens, uma vez que as lesões orais podem servir como indicadoras fundamentalis no diagnostic precoce da doença.

Palavras clave: Lesões aftóides; Etiología; Tratamiento; Diagnóstico. 


\section{Introdução}

As lesões aftoides compreendem um grupo de doenças que apresentam manifestações clínicas variadas e por vezes devastadoras para o paciente. São doenças inflamatórias que possuem diversas etiologias, como infecciosas, autoimunes, traumáticas, neoplásicas ou reações medicamentosas. Podem representar lesões isoladas, ser manifestação local de um distúrbio sistêmico ou a partir do comprometimento local, levar a um acometimento geral do indivíduo (Abida et al., 2022; Bhat et al., 2010).

A identificação da lesão aftoide na cavidade oral pode representar dificuldades quanto ao diagnóstico para o profissional da saúde. A anamnese do paciente com qualquer queixa de lesão da cavidade bucal deve conter o tempo de evolução da doença e presença de sintomatologia como dor, prurido, dificuldade de alimentação ou alterações sistêmicas (Neville, 2009; Sreeja et al., 2015).

O exame físico se inicia com a avaliação geral do paciente, com ênfase no estado nutricional. O profissional deve observar hábitos como mordiscar bochechas ou passar a língua nos lábios. A acurada descrição da lesão é o passo mais importante, incluindo a região acometida e distribuição, lesão única ou múltiplas, consistência da lesão, coloração e características das bordas (Cui et al., 2016).

Diante do exposto, esse estudo objetivou revisar a literatura acerca das lesões vesico-bolhosas da mucosa oral, destacando sua apresentação clínica, diagnóstico e tratamento, bem como a importância de um correto manejo clínico em pacientes que possuem tais lesões na cavidade oral.

\section{Metodologia}

Trata-se de uma revisão narrativa da literatura. A revisão de literatura permite a busca aprofundada dentro de diversos autores e referenciais sobre um tema específico, nesse caso, as lesões aftoides da cavidade oral (Pereira et al., 2018).

A fim de que haja direcionamento na pesquisa, delineou-se como questão norteadora: “Quais são aftoides da cavidade oral, suas apresentações clínicas, diagnóstico e tratamento?"

Para a construção deste artigo, foi realizado um levantamento bibliográfico nas bases de dados SciVerse Scopus, Scientific Eletronic Library Online (Scielo), U.S. National Library of Medicine (PUBMED) e ScienceDirect, com auxílio do gerenciador de referências Mendeley. Dentre os artigos contemplados para a realização do presente estudo, foram considerados aqueles publicados entre os anos de 2010 e 2021.

A estratégia de pesquisa desenvolvida para identificar os artigos incluídos e avaliados para este estudo baseou-se nos descritores contidos na lista dos Descritores em Ciência da Saúde (DeCS) e suas combinações no idioma português e inglês: [(lesões aftóides OR aphthous lesions) AND (mucosa oral OR oral mucosa OR boca OR mouth OR mucosa jugal OR cheek mucosa OR língua OR tongue) AND (manifestações clínicas OR clinical findings OR tratamento OR treatmente OR etiologia OR etiology OR diagnóstico OR diagnosis)]

\subsection{Critérios de inclusão e exclusão}

Considerou-se como critério de inclusão os artigos completos disponíveis na íntegra nas bases de dados citadas, nos idiomas inglês e português, e relacionados com o objetivo deste estudo. Os critérios de exclusão foram artigos incompletos, duplicados, resenhas, estudos in vitro e resumos. 


\subsection{Seleção de estudos}

A estratégia de pesquisa baseou-se na leitura dos títulos para encontrar estudos que investigassem a temática da pesquisa. Caso contemplasse esse primeiro objetivo, posteriormente, os resumos eram lidos e, persistindo na inclusão, era feita a leitura do artigo completo. Quando havia dúvida sobre a inclusão, o artigo era lido por outro autor e, a decisão de inclusão ou exclusão era tomada em consenso.

\section{Resultados e Discussão}

Com base na revisão de literatura feita nas bases de dados eletrônicas citadas, foram identificados 957 artigos científicos, dos quais 198 estavam duplicados com dois ou mais índices. Após a leitura e análise do título e resumos dos demais artigos, outros 692 foram excluídos. Assim, 70 artigos foram lidos na íntegra e, com base nos critérios de inclusão e exclusão, apenas 22 artigos foram selecionados para compor este estudo. O fluxograma com detalhamento de todas as etapas de seleção está na figura 1.

Figura 1 - Fluxograma de identificação e seleção dos estudos.

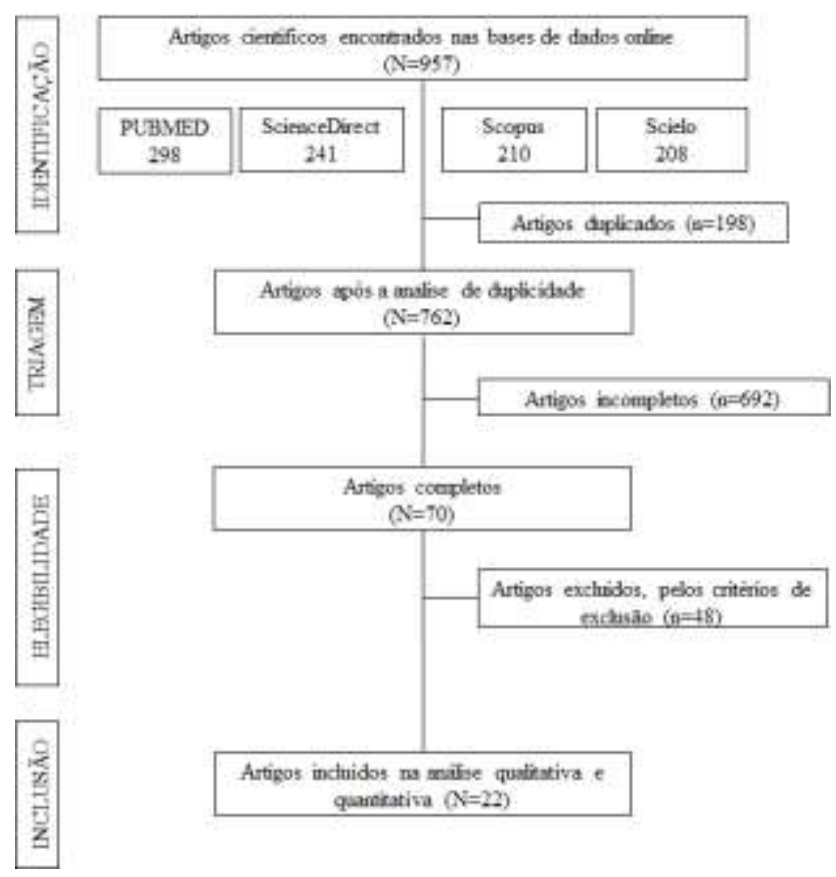

Fonte: Autores (2021).

\subsection{Estomatite aftoide recorrente}

A Estomatite Aftosa Recorrente (EAR) consiste em uma patologia da mucosa oral, que atinge grande parte da população, principalmente indivíduos do sexo feminino. Essas lesões são subclassificadas em três tipos: menor, maior e herpetiforme. A estomatite aftosa recorrente menor é a mais comum entre os três subtipos, possui dimensão menor que 10 milímetros e duração entre 10 a 14 dias. Já as lesões do subtipo maior, são incomuns e mais severas, com duração variando de alguns dias a algumas semanas, resultando, comumente, em cicatrizes locais. Por fim, a forma herpetiforme é a menos comum dentre as três formas, sendo caracterizada por múltiplas e pequenas lesões que se unem, formando úlceras de tamanhos maiores (Miziara et al., 2009; C Scully \& Felix, 2005).

Clinicamente, a EAR é caracterizada por um pródromo de queimação ou dor localizada, geralmente com duração de 24 a 48 horas, que antecede o aparecimento clínico clássico da ulceração, na qual o epitélio é infiltrado por linfócitos. As lesões clássicas da EAR envolvem a mucosa oral não queratinizada, apresentando-se como úlceras rasas redondas ou ovais, dolorosas, 
claramente definidas, com um centro necrótico raso. O próximo passo é o estágio ulcerativo, no qual se desenvolve o edema, acompanhado pela presença de queratinócitos e vasculite superficial localizada, que gera inflamação na região, ulceração e infiltração de plasmócitos, linfócitos e neutrófilos (Gorsky et al., 2007; Suharyani et al., 2021). A resposta imune do hospedeiro leva as células T, macrófagos e mastócitos a liberarem o fator de necrose tumoral alfa (TNF- $\alpha$ ), dando início ao processo de cicatrização (Cui et al., 2016).

As causas da EAR são inúmeras, porém, nenhuma estabelecida de forma precisa. Assim, a etiologia específica da EAR ainda é desconhecida e os pacientes que sofrem desta afecção são, geralmente, indivíduos saudáveis. A maioria das evidências disponíveis na literatura sugere que a EAR consiste em doença inflamatória de caráter não infeccioso com mecanismo imunológico.

Uma vez que a etiologia dessa afecção ainda é desconhecida, não há nenhum protocolo de tratamento estabelecido para a cura das lesões. Assim, o manejo clínico estabelecido é voltado para o alívio dos sintomas ao paciente e melhor cicatrização das úlceras (Da Costa \& Castro, 2013). Medicamentos anti-inflamatórios imunossupressores tópicos, principalmente corticoesteroides tópicos e/ou sistêmicos, bem como analgésicos são as medidas terapêuticas preconizadas (Crispian Scully et al., 2003; Vincent \& Lilly, 1992).

\subsection{Lúpus eritematoso sistêmico}

O Lúpus Eritematoso Sistêmico (LES) é uma doença inflamatória crônica, de caráter autoimune, que acomete múltiplos órgãos, com padrão altamente heterogêneo de manifestações clínicas e sorológicas. A patogênese do LES consiste no resultado das interações entre genes, hormônios e meio ambiente, entretanto, sua etiologia não está bem definida pela literatura (Abida et al., 2022; Carrión-Barberà et al., 2021).

Com relação às características clínicas, o LES possui curso imprevisível, com suas manifestações variando a depender de cada caso. De forma geral, a doença progride em surtos agudos e períodos de remissão, com grandes períodos de atividade inflamatória subclínica. Pode afetar todos os órgãos e sistemas do corpo e, durante as crises, mais de um órgão é geralmente afetado. Nas formas brandas, as articulações e a pele são os principais órgãos afetados. Nas formas moderadas, outros órgãos estão envolvidos, mas é a doença grave, principalmente dos rins e do coração, que pode ser fatal (Abida et al., 2022; Bhat et al., 2010).

Na cavidade oral, as manifestações do LES se apresentam de forma semelhante às lesões do líquen plano erosivo. No entanto, um fator importante que permite a diferenciação dessas duas doenças é o fato de que as lesões do LES raramente ocorrem na ausência de lesões cutâneas, ao contrário do que ocorre em lesões bucais do líquen plano. Assim, em lesões orais causadas peloLES, é notável uma área central eritematosa ulcerada ou atrófica, circundada por estrias brancas finas e irradiadas (Abida et al., 2022; Neville, 2009; Wang et al., 2021).

O tratamento do LES depende do órgão e sistemas envolvidos e da gravidade da doença, o que pode variar de medicamentos tópicos para doenças dermatológicas e anti-inflamatórios não esteroidais para doenças musculoesqueléticas, até imunossupressão sistêmica agressiva (Neville, 2009; Wang et al., 2021).

\subsection{Mucosite}

A mucosite é um processo inflamatório que envolve as membranas mucosas do trato alimentar, ou seja, esôfago, estômago e intestino. A mucosite oral, ulceração dolorosa da mucosa, é o efeito colateral mais comum da radioterapia e/ou quimioterapia, afetando cerca de $70 \%$ dos pacientes com câncer de cabeça e pescoço em tratamento. Nesses pacientes, há comprometimento da qualidade de vida, dificultando até mesmo a administração do próprio esquema antineoplásico, levando à morbidade e mortalidade (Boss et al., 2021; Sonis, 2013). 
A manifestação consiste, nos estágios iniciais da lesão, como uma área de coloração esbranquiçada com ausência de descamação de ceratina. Com a progressão da lesão, há a perda desta camada, com reposição de uma mucosa edemaciada, eritematosa e friável. A evolução da lesão resulta em áreas ulceradas com a presença de membrana superficial fibrinopurulenta amarelada e destacável (Campos et al., 2013). A Organização Mundial da Saúde (OMS) classifica a Mucosite Oral de acordo com seus aspectos clínicos e sintomatologia (Figura 1)

Tabela 1 - Classificação da Mucosite Oral de acordo com a OMS.

\begin{tabular}{cll}
\hline Grau de Mucosite & \multicolumn{1}{c}{ Exame Clínico } & \multicolumn{1}{c}{ Sintomatologia } \\
\hline Grau 0 & Ausente & Ausente \\
Grau 1 & $\begin{array}{l}\text { Presença de eritema } \\
\text { Áreas eritematosas } \\
\text { ulceradas } 2\end{array}$ & $\begin{array}{l}\text { Sintomas mínimos } \\
\text { Paciente suporta } \\
\text { alimentação sólida }\end{array}$ \\
Grau 3 & $\begin{array}{l}\text { Áreas eritematosas } \\
\text { ulceradas }\end{array}$ & e $\begin{array}{l}\text { Paciente com alimentação } \\
\text { líquida } \\
\text { Gecrose e sangramento } \\
\text { espontâneo } 4\end{array}$ \\
& $\begin{array}{l}\text { Paciente não consegue se } \\
\text { alimentar por via oral }\end{array}$ \\
\hline
\end{tabular}

Fonte: Binda et al. (2021).

\section{Considerações Finais}

A partir das trajetórias bibliográficas dos pesquisadores, esse estudo abordou lesões aftóides que acometem a cavidade oral, abordando seus conceitos, características clínicas, diagnóstico e tratamento.

Os resultados desta pesquisa revelam três principais lesões aftoides na mucosa oral, sendo elas estomatite aftosa recorrente, lúpus eritematoso sistêmico e mucosite oral. Diante disso, é de extrema importância que os profissionais da suade estejam familiarizados com essas lesões, distinguindo-as de lesões malignas que afetam a cavidade oral, a afim de que se estabeleça o correto tratamento para cada caso.

Com isso, o levantamento bibliográfico sobre as lesões aftoides da mucosa oral se mostra importante, pois envolve estudos retrospectivos que possuem relação com o tema proposto e, ao mesmo, tempo, cria-se a possibilidade futura de novos pesquisadores abordarem o tema, criando novos trabalhos, para que haja melhor direcionamento quanto ao diagnóstico e tratamento correto dessas lesões.

\section{Referências}

Abida, R., Yeoh, S.-A., \& Isenberg, D. A. (2022). Advances in systemic lupus erythematosus. Medicine, 50(1), 7-17. https://doi.org/https://doi.org/10.1016/j.mpmed.2021.10.003

Bhat, A., Naguwa, S., Cheema, G., \& Gershwin, M. E. (2010). The epidemiology of transverse myelitis. Autoimmunity Reviews, 9(5), A395-A399. https://doi.org/https://doi.org/10.1016/j.autrev.2009.12.007

Binda, N. C., Binda, A. L. C., Rodrigues, S. de A., Loures, L. L. da S., Sá, A. C. S. F. de, Silva, J. V. L., Franco, A. G., Borba, T. O. da S., Magalhães, P. Í. B. M. de A., Araújo, E. L. da S. F. de, Souza, H. Y. M. S. de, Barbosa, R. L. S., Savi, F. da R., Reis, J. L., Ramalho, M. A., Fernandes, C. da S., \& Sátiro, V. D. de S. (2021). Manifestações orais decorrentes da radioterapia de cabeça e pescoço. In Research, Society and Development. $10(12)$, e411101220519. https://doi.org/10.33448/rsd-v10i12.20519

Boss, M.-K., Ke, Y., Bian, L., Harrison, L. G., Lee, B.-I., Prebble, A., Martin, T., Trageser, E., Hall, S., Wang, D. D., Wang, S., Chow, L., Holwerda, B., Raben, D., Regan, D., Karam, S. D., Dow, S., Young, C. D., \& Wang, X.-J. (2021). Therapeutic Intervention Using a Smad7-Based Tat Protein to Treat RadiationInduced Oral Mucositis. International Journal of Radiation Oncology*Biology*Physics. https://doi.org/https://doi.org/10.1016/j.ijrobp.2021.09.039

Campos, L., Carvalho, D. L. C., Castro, J. R., \& Simões, A. (2013). Laserterapia no tratamento da mucosite oral induzida por quimioterapia: relato de caso. Revista Da Associacao Paulista de Cirurgioes Dentistas, 67(2), 102-106.

Carrión-Barberà, I., Salman-Monte, T. C., Vílchez-Oya, F., \& Monfort, J. (2021). Neuropsychiatric involvement in systemic lupus erythematosus: A review. 
Research, Society and Development, v. 11, n. 2, e52111226056, 2022

(CC BY 4.0) | ISSN 2525-3409 | DOI: http://dx.doi.org/10.33448/rsd-v11i2.26056

Autoimmunity Reviews, 20(4), 102780. https://doi.org/https://doi.org/10.1016/j.autrev.2021.102780

Cui, R. Z., Bruce, A. J., \& Rogers, R. S. 3rd. (2016). Recurrent aphthous stomatitis. Clinics in Dermatology, 34(4), 475-481. https://doi.org/10.1016/j.clindermatol.2016.02.020

Da Costa, G. B. F., \& Castro, J. F. L. (2013). Etiologia e tratamento da estomatite aftosa recorrente - Revisão de literatura. Medicina (Brazil), 46(1), 3-9. https://doi.org/10.11606/issn.2176-7262.v46i1p1-7

Gorsky, M., Epstein, J., Rabenstein, S., Elishoov, H., \& Yarom, N. (2007). Topical minocycline and tetracycline rinses in treatment of recurrent aphthous stomatitis: a randomized cross-over study. Dermatology Online Journal, 13(2), 1.

Miziara, I. D., Costa, K. C., Mahmoud, A., Weber, R., Wilhelmsen, N. S. W., \& Imamura, R. (2009). Laryngeal manifestations in atypical Recurrent Aphthous Stomatitis. Brazilian Journal of Otorhinolaryngology, 75(5), 660-664. https://doi.org/10.1590/s1808-86942009000500008

Neville, B. W. D. D. C. A. J. B. (2009). Oral and Maxillofacial Pathology. (3rd ed.).

Pereira, A., Shitsuka, D., Parreira, F., \& Shitsuka, R. (2018). Método Qualitativo, Quantitativo ou Quali-Quanti. In Metodologia da Pesquisa Científica. https://repositorio.ufsm.br/bitstream/handle/1/15824/Lic_Computacao_Metodologia-Pesquisa-Cientifica.pdf?sequence=1. Acesso em: 28 março 2020.

Scully, C, \& Felix, D. H. (2005). Oral medicine--update for the dental practitioner. Aphthous and other common ulcers. British Dental Journal, 199(5), 259264. https://doi.org/10.1038/sj.bdj.4812649

Scully, Crispian, Gorsky, M., \& Lozada-Nur, F. (2003). The diagnosis and management of recurrent aphthous stomatitis: a consensus approach. Journal of the American Dental Association (1939), 134(2), 200-207. https://doi.org/10.14219/jada.archive.2003.0134

Sonis, S. T. (2013). Oral Mucositis in Head and Neck Cancer: Risk, Biology, and Management. American Society of Clinical Oncology Educational Book, 33, e236-e240. https://doi.org/10.14694/EdBook_AM.2013.33.e236

Suharyani, I., Fouad Abdelwahab Mohammed, A., Muchtaridi, M., Wathoni, N., \& Abdassah, M. (2021). Evolution of Drug Delivery Systems for Recurrent Aphthous Stomatitis. Drug Design, Development and Therapy, 15, 4071-4089. https://doi.org/10.2147/DDDT.S328371

Vincent, S. D., \& Lilly, G. E. (1992). Clinical, historic, and therapeutic features of aphthous stomatitis. Literature review and open clinical trial employing steroids. Oral Surgery, Oral Medicine, and Oral Pathology, 74(1), 79-86. https://doi.org/10.1016/0030-4220(92)90219-g

Wang, M., Wang, Z., Zhang, S., Zhang, L., Zhao, J., Wang, Q., Tian, X., Li, M., Chiganer, E. H., \& Zeng, X. (2021). Relapse rates and risk factors for unfavorable neurological prognosis of transverse myelitis in systemic lupus erythematosus: A systematic review and meta-analysis. Autoimmunity Reviews, 102996. https://doi.org/https://doi.org/10.1016/j.autrev.2021.102996 